\title{
LOCAL CONNECTION IN LOCALLY COMPACT SPACES
}

\section{H. A. NEWMAN}

It was proved by Hurewicz ${ }^{1}$ that a compact space which is both $\mathrm{LC}^{1}$ and $\mathrm{lc}^{n}$ is $\mathrm{LC}^{n}$. In the present paper the corresponding result for locally compact spaces is proved, (a) for uniform local connection, and (b) for relative local connection. ${ }^{2}$ The extension of Hurewicz's theorem to locally compact spaces is included in (b). The main diffculty in extending Hurewicz's methods is that his "Satz 6," on the passage from $\epsilon$-homotopy to true homotopy, cannot be carried over to locally compact spaces without substantial modification, even when uniform local connection is assumed. To overcome this a stronger form of the $\mathrm{lc}^{p}$ and $\mathrm{LC}^{p}$ conditions is used, namely (for $\mathrm{Ic}^{p}$ ), the existence of a function $\zeta(\delta, x)$ such that, given a compact set $F$ in the neighbourhood $U(x, \zeta(\delta, x))$ of any point $x$, there is a compact subset $F^{\prime}$ of $U(x, \delta)$ such that every $q$-cycle in $F$ bounds in $F^{\prime}$, for $0 \leqq q \leqq p$; and analogously for LC ${ }^{p}$. It is shown that these are equivalent to the ordinary $\mathrm{lc}^{p}$ and $\mathrm{LC}^{p}$ properties in locally compact (metric) spaces.

1. Definitions. It is assumed once for all that the containing space $X$ is locally compact, and has metric $\rho .^{8}$ Homologies are relative to integral coefficients; cycles in $X$ are Vietoris-cycles (but finite cycles are chains on some simplicial complex with vertices in $X$ ). The statement that $\Gamma$ bounds in $E$ means that $\Gamma$ bounds in a compact subset of $E$. $p$ denotes an integer not less than 0 .

The letter $F$, with various suffixes, always denotes a compact set. If $G$ is open, the statement " $F \subseteq G$ with a margin $\alpha$ " means that $\alpha>0$, and $\mathrm{Cl}(U(F, \alpha))$ is compact and contained in $G .^{4}$ The existence of margins for every such $F$ and $G$ is ensured by the local compactness of $X$.

A set $E_{1}$ is $a c^{p}$ rel. $E_{2}$ ("acyclic up to $p$ rel. $E_{2}$ ") if every $q$-cycle in $E_{1}$ bounds in $E_{2}$, for $0 \leqq q \leqq p$. $E_{1}$ is $a s^{p}$ rel. $E_{2}$ ("aspherical up to $p$ rel. $\left.E_{2}{ }^{n}\right)$ if every mapping of the $q$-sphere $S^{q}$ into $E_{1}$ is null-homotopic in $E_{2}$, for $0 \leqq q \leqq p$. The set $E_{1}$ is strongly ac ${ }^{p}$ (or strongly as ${ }^{p}$ ) rel. $E_{2}$ if, given any $F$ in $E_{1}$ there is an $F^{\prime}$ in $E_{2}$ such that $F$ is ac ${ }^{p}\left(\right.$ or as ${ }^{p}$ ) rel. $F^{\prime}$.

Presented to the Society, July 19, 1948; received by the editors October 25, 1948.

${ }^{1}$ Hurewicz [4], denoted hereafter by $\mathrm{H}$. Numbers in brackets refer to the bibliography at the end of the paper.

2 Problem 4 of Eilenberg and Wilder [ 3 ] is thereby settled affirmatively.

${ }^{3}$ Use is made at one point (Theorem $1,(B)$ ) of a local separability condition.

- Cl. $(X)$ denotes the closure of $X$. 
Among the various definition of $1 c^{p}$ and $u c^{p}$ that are in the field we choose those which impose the heaviest conditions on the bounding cycles, and therefore the lightest conditions on the space. If $E_{1} \subseteq E_{2}$ $\subseteq X, E_{1}$ is $\mathrm{lc}^{p}$ rel. $E_{2}$ if there is a positive function $\eta(x, \delta)$ such that $E_{1} U(x, \eta(x, \epsilon))$ is ac ac $^{p}$ rel. $E_{1} U(x, \epsilon)$ for all points $x$ of $E_{2}$ and any positive $\epsilon ;^{5}$ and $X$ is (absolutely) $l c^{p}$ if it is $l^{p}$ rel. $X$. The space $X$ is ulc $^{p}$ if there is a positive function $\eta(\epsilon)$ such that for all points $x$ of $X, U(x, \eta(\epsilon))$ is ac ${ }^{p}$ rel. $U(x, \epsilon)$.

2. Homology. We consider chain-realisations of (abstract simplicial) complexes, in the sense of Lefschetz [7] and Begle [1]. The complexes realised all have their vertices in $X$, and every realisation $t$ of a complex $K$ is to satisfy $t\left(\sigma^{0}\right)=\sigma^{0}$, for all vertices $\sigma^{0}$ of $K$. If $C$ is a finite chain on a complex in $X$, a realisation of $C$ means a chain $t(C)$, where $t$ is a realisation of the carrier complex $\|C\| .^{6}$

If $X$ is connected, a realisation of any complex can, by an arbitrarily small displacement of the vertices, be so modified that accidental clashes are avoided, that is, any common vertex of $\|t(\sigma)\|$ and $\left\|t\left(\sigma^{\prime}\right)\right\|$ belongs to some $\left\|t\left(\sigma^{\prime \prime}\right)\right\|$, where $\sigma^{\prime \prime}$ is a common sub-cell of $\sigma$ and $\sigma^{\prime}$. It will be assumed that this is always arranged. There is, then, for any vertex $x$ of $\left\|t\left(C^{p}\right)\right\|$, a unique simplex $\sigma$ of lowest dimension in $\left\|C^{p}\right\|$, such that $x \in\|t(\sigma)\|$. This $\sigma$ may be called the $C^{p_{\text {-carrier }}}$ of $x$.

TheOREM 1. If $X$ is $l c^{p}$ and $\alpha>0$, and if $F$ is a compact subset of $X$, there exists a finite set of p-cycles $\Gamma_{1}^{p}, \Gamma_{2}^{p}, \cdots, \Gamma_{k}^{p}$ in $U(F, \alpha)$ such that every $\Gamma^{p}$ in $F \sim \sum_{i=1}^{k} n_{i} \Gamma_{i}^{k}$ in $U(F, \alpha)$, for suitable integers $n_{i}{ }^{7}$

Since $X$ is 0 -lc its components are open sets, and therefore the compact set $F$ meets only a finite number of them. It is clearly sufficient to prove the theorem for each separate component meeting $F$, that is, we may assume $X$ to be connected.

The theorem is proved by combining the following results.

(A). Given a compact set $F_{1}$ there exists a positive function $\lambda(\epsilon)$

6 Cf. Eilenberg and Wilder [3] for the corresponding homotopy property.

- Note that the definition of a partial realisation $t$ of $K$ requires $t\left(\sigma^{0}\right)$ to be defined for all 0 -cells $\sigma^{0}$ of $K$. The norm of a full or partial realisation $t$ of $K$ is $\max \rho(x, y)$ for $x \in\left\|t\left(\sigma_{i}\right)\right\|, y \in\left\|t\left(\sigma_{i}\right)\right\|$, where $\sigma_{i}$ and $\sigma_{i}$ are subcells of the same cell $\sigma$ of $K$. The mesh of $t$ is $\max \Delta\left\|\sigma^{\prime}\right\|$ for all simplexes $\sigma^{\prime}$ of chains $t(\sigma)$. ( $\Delta E=$ diameter of $E$.)

7 Wilder [8] (see also Begle [2, Corollary 2.3]) has proved that when homologies are $\bmod m$, the conditions of Theorem 1 imply that at most a finite number of cycles of $F$ are independent in $U(F, \alpha)$. The analogous result with integral coefficients is not strong enough for present purposes, since it would allow, for example, an infinite base $\left(\Gamma_{i}^{p}\right)$ with $\Gamma_{i}^{p} \sim 2 \Gamma_{i+1}^{p}$. 
such that any partial chain-realisation $t_{0}$ of a complex of dimension not greater than $p+1$ of norm less than $\lambda(\epsilon)$ in $F_{1}$ can be extended to a full realisation in $X$ of norm less than $\epsilon$; and there is a positive function $\kappa(\delta, \epsilon)$ such that if mesh $t_{0}<\kappa(\delta, \epsilon)$, mesh $t$ can be made less than $\delta$.

This is Begle [2], Theorem 2.1.8 (Note that when $t_{0}$ is defined only for vertices the $k$-condition is automatically satisfied.)

The $\alpha$ of the enunciation of Theorem 1 may be supposed such that $\mathrm{Cl}(U(F, \alpha))$ is compact. Let $\sum_{0}^{\infty} \epsilon_{n}$ be a positive series with sum less than $\alpha$, such that $\epsilon_{n+1}<\epsilon_{n} / 3$ and, taking $F_{1}=\mathrm{Cl}(U(F, \alpha))$ in (A), $\epsilon_{n+1}<\lambda\left(\epsilon_{n}\right)$.

(B) Every finite cycle $C_{0}^{p}$ in $F$ of mesh less than $\epsilon_{1}$ is the first member of a projection-cycle $\left\{C_{n}^{p}\right\}$ in $U(F, \alpha)$, the projection $\phi_{n}: C_{n+1}^{p} \rightarrow C_{n}^{p}$ being an $\epsilon_{n}$-projection. ${ }^{9}$

Let $\eta_{n}=\sum_{0}^{n} \epsilon_{r}$, and make the inductive hypothesis that a finite cycle $C_{n}^{\eta}$ of mesh less than $\epsilon_{n+1}$ is defined in $U\left(F, \eta_{n-1}\right)$ (for $n=0$, in $F$ ). Since $U\left(F, \eta_{n-1}\right) \subseteq U(F, \alpha) \subseteq F_{1}$, there is by (A) a chain-realisation $C_{n+1}^{p}$ of $C_{n}^{p}$, of mesh less than $\epsilon_{n+2}$ and norm less than $\epsilon_{n}$, and hence contained in $U\left(F, \eta_{n-1}+\epsilon_{n}\right)=U\left(F, \eta_{n}\right)$. This justifies the recursive definition of $C_{n}^{p}$. For each vertex $x$ of $C_{n+1}^{p}$ take $\phi_{n}(x)$ to be any vertex of the $C_{n}^{p}$-carrier of $x$ (defined above). Then $\phi_{n}$ is an $\epsilon_{n}$-projection.

Let the sequence $\left(\epsilon_{n}\right)$ satisfy the conditions of $(B)$, and also $\epsilon_{n+3}<\kappa\left(\epsilon_{n+2}, \epsilon_{n}\right)$ ( $F_{1}$ being as before).

(C) Let $\Gamma_{r}^{p}=\left(Z_{r n}^{p}\right)$ be, for $r=1,2, a$-cycle in $X$. Sufficient conditions for $\Gamma_{1}^{p} \sim \Gamma_{2}^{p}$ in $U(F, \alpha)$ are

$$
Z_{r n}^{p} \sim_{\epsilon_{n+2}} Z_{r, n+1}^{p} \text { in } U\left(F, \eta_{n}\right) \quad \text { for } n \geqq 0
$$

and

$$
Z_{10}^{p} \sim_{\epsilon_{2}} Z_{20}^{p} \text { in } U\left(F, \epsilon_{0}\right) .
$$

Let $Z_{r, n+1}^{p}-Z_{r, n}^{p}=\beta Y_{m n}^{p+1}$ (=boundary of $Y_{m}^{p+1}$ ), where $Y_{m}^{p+1}$ is a chain of mesh less than $\epsilon_{n+3}$ in $U\left(F, \eta_{n}\right)$ (whence mesh $\left.Z_{m}^{p}<\epsilon_{n+3}\right)$; and let $Z_{10}^{p}-Z_{20}^{p}=\beta D_{0}^{p+1}$, where $D_{0}^{p+1}$ is of mesh less than $\epsilon_{2}$, and $D_{0}^{p+1}$ $\subseteq U\left(F, \epsilon_{0}\right)$. Assume inductively that for some $n \geqq 0$, a finite chain $D_{n}^{p+1}$ has been defined so that $\beta D_{n}^{p+1}=Z_{1 n}^{p}-Z_{2 n}^{p}$, mesh $D_{n}^{p+1}<\epsilon_{n+2}$, and $D_{n}^{p+1} \subseteq U\left(F, \eta_{n}\right)$. Then $Q_{n}^{p+1}=D_{n}^{p+1}+Y_{1 n}^{p+1}-Y_{2 n}^{p+1}$ is a $(p+1)$-chain with boundary $Z_{1, n+1}^{p}-Z_{2, n+1}^{p}$, and $\left\|Q_{n}^{p+1}\right\| \subseteq U\left(F, \eta_{n}\right) \subseteq F_{1}$. A partial realisation, $t_{0}$, of $\left\|Q_{n}^{p+1}\right\|$ is determined by putting $t_{0}(\sigma)=\sigma$ if ${ }^{10} \sigma \in\left\|Q_{n}^{0}\right\|$

- Our definitions are slightly different, but the proof is almost exactly similar.

- Cf. Begle [1, Lemma 2.4]. The property asserted of $\phi_{n}$ means that the projection-prism has mesh less than $\epsilon_{n}$, and hence mesh $C_{n}^{p}<\epsilon_{n+1}$.

${ }^{10}\left\|Q_{m}^{0}\right\|=$ set of vertices of $\left\|Q^{p+1}\right\|$, and in general $K^{m=}$ set of cells of $K$ of dimensions not greater, than $m$. 
$\cup\left\|Z_{1, n+1}^{p}\right\| \cup\left\|Z_{2, n+1}^{p}\right\|$. Mesh $t_{0}<\epsilon_{n+4}<\kappa\left(\epsilon_{n+3}, \epsilon_{n+1}\right)$, and norm $t_{0} \leqq$ mesh $\left\|\phi_{n}^{p+1}\right\|<\epsilon_{n+2}<\lambda\left(\epsilon_{n+1}\right)$. Hence $t_{0}$ can be extended to give a realisation $D_{n+1}^{p+1}$ of $Q_{n}^{p+1}$, of mesh less than $\epsilon_{n+3}$, and norm less than $\epsilon_{n+1}$. Hence

$$
D_{n+1}^{p+1} \subseteq U\left(F, \eta_{n}+\epsilon_{n+1}\right)=U\left(F, \eta_{n+1}\right) ;
$$

and

$$
\beta D_{n+1}^{p+1}=t\left(\beta Q_{n}^{p+1}\right)=t\left(Z_{1, n+1}^{p}-Z_{2, n+1}^{p}\right)=Z_{1, n+1}^{p}-Z_{2, n+1}^{p} .
$$

The recursive definition of $D_{n}^{p+1}$ is justified, and (C) is therefore proved.

Proof of Theorem 1. Let $\left\{U\left(x_{i}, \epsilon_{4} / 6\right\}\right.$ be a finite covering of $F$, with $x_{i} \in F$, and let $N$ be the nerve of the covering $\left\{U\left(x_{i}, \epsilon_{4} / 2\right)\right\}$, with the points $x_{i}$ as vertices. Choose a $p$-dimensional basis of homology $C_{(i)}^{p}(i=1,2, \cdots, k)$ of $N$. Since mesh $N<\epsilon_{4},(\mathrm{~B})$ is applicable, with the series $\left(\epsilon_{0}, \epsilon_{1}, \cdots\right)$ replaced by $\left(\epsilon_{3}, \epsilon_{4}, \cdots\right)$, to give a projection-cycle $\Gamma_{i}^{p}$ in $U(F, \alpha)$ with first member $C_{(i)}^{p}$. The cycles $\Gamma_{i}^{p}$ are the required set. For let $\Gamma^{p}$ be any $p$-cycle in $F$, and $\left(Z_{n}^{p}\right)$ $(n=0,1, \cdots)$ a subsequence of its members satisfying

$$
Z_{n+1}^{p} \sim_{\iota_{n+3}} Z_{n}^{p} \text { in } F,
$$

$\operatorname{mesh} Z_{0}^{p}<\epsilon_{4} / 6$.

If, for each $x$ of $F, \theta(x)$ is a vertex of $N$ in $U\left(x, \epsilon_{4} / 6\right)$, and if $x$ and $y$ are vertices of the same cell of $Z_{0}^{p}, \rho(\theta x, \theta y)<\epsilon_{4} / 6+\rho(x, y)+\epsilon_{4} / 6$ $<\epsilon_{4} / 2$, and hence $\theta$ is a simplicial mapping of $Z_{0}^{p}$ into $N$.

Let $\theta\left(Z_{0}^{p}\right)=Z^{p} \sim \sum_{1}^{k} n_{i} C_{(i)}^{p}$ in $N$, for some $n_{i}$. The pair of cycles $\Gamma^{p}$ and $\sum_{1}^{k} n_{i} \Gamma_{i}^{p}$ satisfy the conditions (1) and (2) of (C). Let $\Gamma_{i}^{p}$ $=\left\{C_{0 i}, C_{1 i}, C_{2 i}, \cdots\right\}$. Condition (1). For $\Gamma^{p}$ this follows from (a) above. The chain $\sum n_{i} \Gamma_{i}^{p}$ is a projection-cycle for which $\phi_{n}$ is an $\epsilon_{n+3}$-projection, and all vertices of $\left\|\sum n_{i} C_{n i}\right\|$ belong to $\left\|\sum n_{i} C_{n+1, i}\right\| .{ }^{11}$ Hence condition (1) is satisfied if $\left\|C_{n+1, i}\right\| \subseteq U\left(F, \eta_{n}\right)$. This is so, since $\left\|C_{n+1, i}\right\| \subseteq U\left(F, \sum_{3}^{n+3} \epsilon_{r}\right)$ (proof of $(\mathbf{B}), \epsilon_{r+3}$ replacing $\epsilon_{r}$ ), and $\sum_{3}^{n+3} \epsilon_{r}<\sum_{0}^{n} \epsilon_{r}=\eta_{n}$. Condition (2). The $\theta$-prism joining to $Z_{0}^{p} Z^{\prime p}$ has mesh less than $\epsilon_{4} / 2<\epsilon_{2}$, whence $Z_{2}^{p} \sim_{\epsilon_{2}} Z^{\prime p}$ in $F$; and $Z^{\prime p} \sim \sum n_{i} C_{(i)}^{p}$ in $N$, a complex of mesh less than $\epsilon_{2}$ in $F$.

TheOREM 2. If $X$ is $l c^{p}$, an open set $G_{1}$ which is ac rel. an open set $G_{2}$ is also strongly ac rel. $G_{2}$.

Suppose $F \subseteq G_{1}$ with a margin $\alpha$. For $0 \leqq q \leqq p$, let $\Gamma_{1}^{q}, \Gamma_{2}^{q}, \cdots, \Gamma_{\boldsymbol{k}_{q}}^{q}$ be a basis of $q$-cycles in $U(F, \alpha)$ constructed as in Theorem 1. Let $\Gamma_{\text {? }}^{q}$

"By the general rule that $t\left(\sigma^{0}\right)=\sigma^{0}$, above. 
bound in the subset $F_{q i}$ of $G_{2}$. Then every $q$-cycle in $F$ bounds in the compact subset

$$
F^{\prime}=\mathrm{Cl}(U(F, \alpha)) \cup \bigcup_{q=0}^{p} \bigcup_{i=1}^{k_{q}} F_{q i}
$$

of $G_{2}$.

3. Homotopy. The relation of homotopy is denoted by $\simeq$ and $\epsilon$-homotopy ${ }^{12}$ by $\simeq_{c} . S^{p}$ is the sphere $\sum_{0}^{p} \xi_{r}^{2}=1$ in $R^{p+1} ; S^{r}$ is, for $r<p$, the intersection of $S^{r+1}$ with $\xi_{r+1}=0 ; c_{0}$ is the point $(1,0, \cdots, 0)$ of $R^{p+1}$. A set $E_{1}$ is $\epsilon$-as $^{p}$ rel. $E_{2}$ if, for $0 \leqq q \leqq p$, every mapping $f: S^{q \rightarrow} E_{1} \simeq_{6} 0$ in $E_{2}$ rel. $c_{0}$.

Theorem 3. Let the open set $G$ be $L C^{p-1}$ rel. $X^{18}$ and suppose that a positive function $\eta_{0}(\delta, x)$ exists with this property: to any point $x$, and any compact $F$ in $G U\left(x, \eta_{0}(\delta, x)\right)$ there corresponds a compact $F^{\prime}$ in $G U(x, \delta)$ such that $F$ is $\epsilon$-as ${ }^{p}$ rel. $F^{\prime}$ for every positive $\epsilon$. Then $G U\left(x, \eta_{0}(\delta, x)\right)$ is strongly as ${ }^{p}$ rel. $G U(x, \delta)$; and therefore $G$ is $L C^{p}$ rel. $X$. (The $L C^{p-1}$ condition is vacuously satisfied if $p=0$.)

Corollary. If $G=X$ and $\eta_{0}(\delta, x)=\eta_{0}(\delta)$, independent of $x, X$ is $p-U L C$.

This theorem replaces "Satz 6" of Hurewicz [4] in locally compact spaces. Although our proof follows his closely, the many changes of detail make it necessary to give the full proof, which depends on the following lemmas $\mathrm{D}, \mathrm{E}$, and $\mathrm{F}$.

(D) ( $p \geqq 1)$ Given a compact set $F$ in a locally compact $L C^{p-1}$ space $X$, and a positive number $\epsilon$, there exists a positive $\eta_{2}=\eta_{2}(\epsilon, F)$ with the following property: if $P$ is a polyhedron, and $Q$ a subpolyhedron ${ }^{14}$ of $P^{p-1}$, and if $f_{0}, f_{1}$ map $P$ into $F$ and satisfy $\rho\left(t_{0}, t_{1}\right)<\eta_{2}$, there exists $a$ mapping $f_{0}^{\prime}: P \rightarrow X$, agreeing with $f_{0}$ on $Q$, and deformable into $f_{1}$ within $\epsilon$, in $X .15$

The proof of (D) is omitted, since only obvious changes are needed in the proofs of $\mathrm{H}$, Sätze 1-3.

Let $G$ be as in Theorem 3. Let $F_{0} \subseteq G$ with a margin $\alpha_{0}$, and let $0<\delta \leqq \alpha_{0}$. There exists a finite covering $\left\{U_{i}\right\}=\left\{U\left(x_{i}, \eta_{0}\left(\delta, x_{i}\right)\right) / 2\right\}$

12 See H, p. 477: $f_{1} \sim_{\epsilon} f_{2}$ if $f_{1}$ and $f_{2}$ are connected by an $\epsilon$-chain of points in the space of mappings.

${ }^{13}$ This is the homotopy-local-connection introduced by Lefschetz [5]. For relative local connection see Eilenberg and Wilder [3].

14 No distinction is made in terminology between a polyhedral complex $P$ and the polyhedron which is its locus; but the corresponding abstract simplicial complex determined by the vertices of $P$ is to be distinguished from $P$. It is denoted by $\|P\|$.

1s That is, $f_{0}^{\prime}$ and $f_{1}$ are connected in the space $X^{P}$ by an arc of diameter less than $\epsilon$. 
of the compact set $F_{0}$, with $x_{i} \in F_{0}$. Since $\eta_{0}\left(\delta, x_{i}\right) \leqq \delta \leqq \alpha_{0}, U U_{i} \subseteq G$. Let $\eta_{3}=\min _{i} \eta_{0}\left(\delta, x_{i}\right)$.

(E) ( $p \geqq 1)$ If $f_{1}, f_{2}$ map the p-element $E^{p}$ (with boundary $S^{p-1}$ ) into $F_{0}$, and if $f_{1}\left|S^{p-1}=f_{2}\right| S^{p-1}$ and $\Delta f_{r}\left(E^{p}\right)<\eta_{3} / 2$, for $r=1,2$, then $f_{1} \simeq_{6} f_{2}$ with fixed $S^{p-1}$, in $U\left(x_{i}, \delta\right)$, for some $i$.

If $y \in f_{1}\left(S^{p-1}\right)$, one of the points $x_{i}$ satisfies $\rho\left(x_{i}, y\right)<\eta_{0}\left(\delta, x_{i}\right) / 2$ and therefore $f_{r}\left(E^{p}\right) \subseteq U\left(x_{i}, \eta_{3} / 2+\eta_{0}\left(\delta, x_{i}\right) / 2\right) \subseteq U\left(x_{i}, \eta_{0}\left(\delta, x_{i}\right)\right)$ for $r=1,2$. Hence $f_{1} \simeq_{\epsilon} f_{2}$ in $U\left(x_{i}, \delta\right)$, by the conditions of Theorem 3 and H, Satz 4.

We suppose that for each compact $F_{0}$ and each positive $\delta$, such a covering $\left\{U_{i}\right\}$ is chosen, and denote the corresponding $\min _{i} \eta_{0}\left(\delta, x_{i}\right)$ by $\eta_{3}\left(\delta, F_{0}\right)$. Let $\mu(x, y)$ be defined as in H. ${ }^{16}$

(F) Given any compact $F_{0}$ in $G$ there exists $\eta_{4}\left(\delta, F_{0}\right)>0$ such that if $f_{1}, f_{2}$ map $S^{p}$ into $F_{0}$ and $\rho\left(f_{1}, f_{2}\right)<\eta_{4}\left(\delta, F_{0}\right)$, then $\mu\left(f_{1}, f_{2}\right)<\delta$. (The distances $\rho\left(f_{1}, f_{2}\right)$ and $\mu\left(f_{1}, f_{2}\right)$ are in the function space $X^{s^{p}}$.)

First suppose $p>0$. Let $\alpha$ be a margin of $F_{0}$ rel. $G$. We define:

$$
\begin{array}{rlrl}
\delta^{\prime} & =\min (\delta, \alpha), & & F_{1}=\mathrm{Cl}\left(U\left(F_{0}, \alpha\right)\right), \\
\xi=\frac{1}{6} \eta_{3}\left(\frac{1}{4} \delta^{\prime}, F_{1}\right), & \eta_{4}\left(\delta, F_{0}\right)=\eta_{2}\left(\xi, F_{0}\right),
\end{array}
$$

the functions $\eta_{2}, \eta_{3}$ having the meanings given above. Let two mappings $f_{1}, f_{2}$ of $S^{p}$ into $F_{0}$ be given, satisfying $\rho\left(f_{1}, f_{2}\right)<\eta_{4}\left(\delta, F_{0}\right)$. Let $S^{p}$ be simplicially subdivided into a polyhedron $\Sigma^{p}$, so finely that $\Delta f_{r}\left(\sigma^{p}\right)<\xi$ for each (continuous) $p$-simplex $\sigma^{p}$ of $\Sigma^{p}, r=1,2$. Then by (D), a mapping $f_{1}^{\prime}: S^{p} \rightarrow X$ exists, agreeing with $f_{1}$ on $\Sigma^{p-1}$, and such that $f_{2} \simeq f_{1}^{\prime}$ within $\xi$ in $G$. Hence $\mu\left(f_{1}^{\prime}, f_{2}\right)<\xi<\delta / 2$. Also, since $\delta^{\prime} \leqq \alpha$ $f_{1}^{\prime}\left(S^{p}\right) \subseteq U\left(F_{0}, \alpha / 2\right) \subseteq F_{1}$; and $\rho\left(f_{1}^{\prime}, f_{2}\right) \leqq \mu\left(f_{1}^{\prime}, f_{2}\right)<\xi$. Therefore $\Delta f_{1}^{\prime}\left(\sigma^{p}\right)$ $<3 \xi=\eta_{3}\left(4^{-1} \delta^{\prime}, F_{1}\right) / 2$ for every $\sigma^{p}$ of $\Sigma^{p}$. By Lemma (E) it follows that $f_{1}^{\prime}\left|\sigma^{p} \simeq_{f} f_{1}\right| \sigma^{p}$, with fixed $\beta \sigma^{p}$, in a set of diameter $\delta / 2$, for every positive $\epsilon$. Since this holds for every $\sigma^{p}$ of $\Sigma^{p}, \mu\left(f_{1}^{\prime}, f_{1}\right)<\delta / 2$. Thus $\mu\left(f_{1}, f_{2}\right) \leqq \mu\left(f_{1}, f_{1}^{\prime}\right)+\mu\left(f_{1}^{\prime}, f_{2}\right)<\delta$.

If $p=0$ let $\eta_{4}\left(\delta, F_{0}\right)=\eta_{3}\left(\delta^{\prime}, F_{0}\right) / 2$. (The definition of $\eta_{3}$ remains significant when $p=0$.) It is sufficient in this case to show that if $x, y \in F_{0}$ and $\rho(x, y)<\eta_{4}\left(\delta, F_{0}\right)$, then $x$ and $y$ are joined by an $\epsilon$-chain of points of $G$, of diameter less than $\delta$. There exists a point $x_{i}$ such that $\rho\left(x, x_{i}\right)<\eta_{0}\left(\delta^{\prime}, x_{i}\right) / 2$. Since $\rho(x, y)<\eta_{3} / 2 \leqq \eta_{0}\left(\delta^{\prime}, x_{i}\right) / 2, x$ and $y$ are both in $U\left(x_{i}, \eta_{0}\left(\delta^{\prime}, x_{i}\right)\right) \subseteq U\left(x_{i}, \alpha\right) \subseteq G$. Hence the required chain exists, by the conditions of Theorem 3 .

Theorem 3 can now be proved. Let a positive $\delta$, a point $x$ of $X$, and a compact $F$ in $G U\left(x, \eta_{0}(\delta, x)\right)$ be given, and let $F^{\prime}$ be a set as in the

${ }^{16}$ In any metric space $R, \mu(x, y)$ is the greatest lower bound of diameters of subsets of $R$ in which $x$ and $y$ are $\epsilon$-connected for every positive $\epsilon$. 
enunciation of Theorem 3. Then $F$ is as $s^{p}$ rel. $F_{2}=\mathrm{Cl}\left(U\left(F^{\prime}, \beta\right)\right)$, where $\beta$ is any margin of $F^{\prime}$ rel. $G U(x, \delta)$. Let $\delta_{n}=\eta_{4}\left(\beta / 2^{n}, F_{2}\right)$, let $x_{0}$ be a point of $F$, and let $f$ map $S^{p}$ into $F$. By the conditions of the theorem, the points $f$ and $\left[x_{0}\right]$ (=constant function $x_{0}$ ) of $F^{S^{p}}$ are joined by a $\delta_{1}$-chain, $L_{1}$, of points, all lying in $F^{\prime s^{p}}$. Assume inductively that $L_{1}, \cdots, L_{n}$ have been determined, $L_{r}$ being formed by joining each consecutive pair of points of $L_{r-1}$ by a $\delta_{r}$-chain; and that the mappings which are the "points" of $L_{n}$ all map $S^{p}$ into $U\left(F^{\prime}, \sum_{1}^{n-1} \beta / 2^{r}\right)$ $\subseteq F_{2}$ (into $F^{\prime}$ when $n=1$ ). Two consecutive points $f_{r}, f_{s}$ of $L_{n}$ satisfy $\rho\left(f_{r}, f_{s}\right)<\delta_{n}=\eta_{4}\left(\beta / 2^{n}, F_{2}\right)$; and hence by Lemma (F), $\mu\left(f_{r}, f_{s}\right)<\beta / 2^{n}$, that is, $f_{r}$ and $f_{c}$ can be joined by a $\delta_{n+1}$-chain of total diameter less than $\beta / 2^{n}$ in the function space. This justifies the recursive definition of $L_{n}$.

It now follows, exactly as in $\mathrm{H}, \mathrm{p} .481$, that $\mathrm{Cl}\left(\mathrm{U}_{1}^{\infty} L_{n}\right)$ is the locus of a continuous path joining $f$ to $\left[x_{0}\right]$ in $F_{2}^{\$ p}$. The proof of Theorem 3 is thus completed.

4. Homology and homotopy. Theorem 4, general case $(p \geqq 2)$. If $G$ is $L C^{1}$ rel. $X$ and $l c^{p}$ rel. $X$ there is a positive function $\zeta(\delta, x)$ such that $G U(x, \zeta(\delta, x))$ is strongly as ${ }^{p}$ rel. $G U(x, \delta)$ for all $x$ of $X$.

Case 0: Put $p=0$ and omit " $L C^{1}$ rel. $X$ and."

Case 1: Put $p=1$ and omit "and lci $r e l . ~ X . "$

Immediate corollaries of this theorem are

THEOREM $4.1(p \geqq 2)$. If $G$ is $L C^{1}$ and $l c^{p}$ it is $L C^{p}$, all rel. $X$.

(When $G=X$ this is the generalisation of Hurewicz's theorem to locally compact spaces.)

TheOREM $4.2(p \geqq 0)$. If $G$ is $L C^{p}$ rel. $X$ there exists a positive function $\zeta(\delta, x)$ such that $G U(x, \zeta(\delta, x))$ is strongly as ${ }^{p}$ rel. $G U(x, \delta)$ for all $x$ of $X$.

Proof of Theorem 4, Case 0 . Let $\eta(\delta, x)$ be such that $G U(x, \eta(\delta, x))$ is ac ${ }^{0}$ rel. $G U(x, \delta)$, and let $F \subseteq G U(x, \eta(\delta, x))$. By Theorem 2 there is an $F^{\prime}$ in $G U(x, \delta)$ such that $F$ is ac ${ }^{0}$ rel. $F^{\prime}$. This implies that for any positive $\epsilon$ any two points of $F$ are joined by an $\epsilon$-chain in $F^{\prime}$, that is, that $F$ is $\epsilon$-as ${ }^{0}$ rel. $F^{\prime}$ for every positive $\epsilon$. Thus the conditions of Theorem $3(0)$ are satisfied, if $\eta_{0}$ is replaced by $\eta$.

Proof of Theorem 4, CASE 1. This is contained in the following theorem.

Theorem 5. If $X$ is $L C^{1}$, any open set $G_{1}$ which is as ${ }^{1}$ rel. an open set $G_{2}$ is also strongly as ${ }^{1}$ rel. $G_{2}$. 
Let $F \subseteq G_{1}$ with margin $\alpha_{0}$. Then there exist positive $\alpha_{1}$ and $\alpha_{2}$ such that, for $r=0,1$, if $x \in F, U\left(x, \alpha_{r+1}\right)$ is as ${ }^{1}$ rel. $U\left(x, \alpha_{r} / 3\right)$. Let $\left\{U\left(x_{i}, \alpha_{2} / 6\right)\right\}$ be a finite covering of $F\left(x_{i} \in F\right)$, and let $N$ be the nerve of the covering $\left\{U\left(x_{i}, \alpha_{2} / 2\right)\right\}$ realised in $X$, with the $x_{i}$ as vertices. If then $P$ is a polyhedron abstractly isomorphic with $N$, the mapping $g_{0}$ of $P^{0}$ into $N^{0}$ determined by the isomorphism can be extended to a mapping $g_{1}$ of $P^{1}$ into $U\left(F, \alpha_{1} / 3\right)$.

Let $f$ be a re-entrant path in $F$, that is, $f:\langle 0,1\rangle \rightarrow F$ with $f(0)=f(1)$ $=x_{0}=g_{0}\left(z_{0}\right)$, say; and let the points $0<\tau_{1}<\tau_{2}<\cdots<\tau_{k}=1$ divide $f$ into sub-paths $s_{j}$ of diameter less than $\alpha_{2} / 6$. Now each $f\left(\tau_{j}\right)$ is in some $U\left(y_{j}, \alpha_{2} / 6\right)$, where $y_{j} \in N^{0}\left(y_{0}=y_{k}=x_{0}\right)$, and a path $\gamma_{j}$ of diameter less than $\alpha_{1} / 3$ therefore runs from $y_{j}$ to $f\left(\tau_{j}\right)$. By the usual process of extruding "tails" the path $f=\sum s_{j}$ is deformable in $U(F$, $\left.\alpha_{1} / 3\right)$ into

$$
\sum_{j=1}^{k}\left(\gamma_{j-1}+s_{j}-\gamma_{j}\right)
$$

the $\sum$ and + -signs denoting the usual path-summation. Now $\rho\left(y_{j-1}, y_{j}\right)<\alpha_{2} / 6+\Delta s_{j}+\alpha_{2} / 6<\alpha_{2} / 2$ and therefore $y_{j-1}, y_{j}$ are the $g_{1-}$ images of the ends of a 1-cell $\sigma_{j}^{\prime}$ of $P^{1}$. Since $g_{1}\left(\sigma_{j}^{\prime}\right)$ and $\gamma_{j-1}+s_{j}-\gamma_{j}$ are both in $U\left(y_{j}, \alpha_{1}\right)$, the path $\gamma_{j-1}+s_{j}-\gamma_{j}$ is deformable in $U\left(y_{j}, \alpha_{0}\right)$, with fixed end points, into the path $g_{1} \mid \sigma_{j}^{\prime}$. Thus $f \simeq g_{1}(s)$ in $U\left(F, \alpha_{0}\right)$, where $s$ is a path on $P$ with $s(0)=s(1)=z_{0}$.

Let $P_{1}, P_{2}, \cdots, P_{l}$ be the components of $P$, and let the paths $a_{1 r}, a_{2 r}, \cdots, a_{m_{r} r}$ in $P_{r}^{1}$ be representatives of a base of the fundamental group of $P_{r}^{1}$. The path $s$ lies in one component, say $P_{r}^{1}$, and

$$
g_{1} \simeq \simeq g_{1} a_{n_{1} r} \pm g_{1} a_{n_{2} r} \pm \cdots \pm g_{1} a_{n_{\mathbf{h}} r}
$$

on $g_{1}\left(P_{r}^{1}\right) \subseteq U\left(F, \alpha_{1}\right) \subseteq G_{1}$. By hypothesis, for each $i$ and $r, g_{1} a_{i r} \simeq 0$ in $G_{2}$, and therefore in a compact set $F_{i r}$ in $G_{2}$. Hence

$$
f \simeq 0 \text { in } \operatorname{Cl}\left(U\left(F, \alpha_{0}\right)\right) \cup \bigcup_{1}^{l} \bigcup_{1}^{m_{r}} F_{i r}
$$

a compact subset of $G_{2}$ independent of $f$.

Proof of Theorem 4, GeNeral CASE $(p \geqq 2)$. We make the inductive assumption that $4(p-1)$ is proved, and may therefore, by $4.1(p-1)$ and $4.2(p-1)$, assume that $G$ is $\operatorname{LC}^{p-1}$ rel. $X$. Let $\zeta^{\prime}(\delta, x)$ be the function corresponding to $\zeta$ in the dimension $p-1$, and let $\eta(\delta, x)$ be such that $G U(x, \eta(\delta, x))$ is ac ${ }^{p}$ rel. $G U(x, \delta)$ for every $x$. Then $\zeta(\delta, x)$ may be put equal to $\eta\left(\zeta^{\prime}(\delta, x), x\right)$. This choice will be justified by Theorem 3 if it is shown that the condition of that theorem is satisfied, with $n_{0}$ replaced by $\zeta$. 
Let $F \subseteq G U(x, \zeta(\delta, x))$. By Theorem 2, there is an $F_{1}$ $\subseteq G U\left(x, \zeta^{\prime}(\delta, x)\right.$ ) (with a margin $\alpha$ ) such that $F$ is ac ${ }^{p}$ rel. $F_{1}$; and by Theorem $4(p-1)$ there is an $F^{\prime} \subseteq G U(x, \delta)$ such that $\mathrm{Cl}\left(U\left(F_{1}, \alpha\right)\right)$ is as $^{p-1}$ rel. $F^{\prime}$. This is the set $F^{\prime}$ required in Theorem 3.

Since $X$ is $\mathrm{LC}^{p-1}$ and $F_{1}$ is compact, there is ${ }^{17}$ a positive function $\eta_{1}(\xi)$ such that, given a polyhedron $P^{p}$, and any subpolyhedron $Q$ containing all its vertices, any mapping $f_{0}: Q \rightarrow F_{1}$ whose continuous norm ${ }^{18}$ is less than $\eta_{1}(\xi)$ can be extended to a mapping $f_{1}: P^{p} \rightarrow X$ of continuous norm less than $\xi$. Let a mapping $f: S^{p} \rightarrow F$ and a positive $\epsilon<\alpha$ be given. Divide $S^{p}$ simplicially into a polyhedron $\Sigma^{p}$, and let $C^{p}$ be a fundamental $p$-cycle on $\|\Sigma p\|{ }^{14}$ The simplicial division is to be so fine that (a) $\Delta f(\sigma)<\eta_{1}(\epsilon / 2)$ for every (continuous) simplex $\sigma$ of $\Sigma^{p}$; and (b) there exist an abstract complex $K^{p+1}$ containing $\left\|\Sigma^{p}\right\|$ as a subcomplex, a chain $C^{p+1}$ on $K^{p+1}$ with boundary $C^{p}$, and a mapping $f_{1}: K^{p+1} \rightarrow F_{1}$ of mesh less than $\eta_{1}(\epsilon / 2)$, with $f_{1}\left|\Sigma^{0}=f\right| \Sigma^{0}$. That this is possible follows from the definition of $F_{1}$. Let $P^{p+1}$ be a polyhedron such that $\left\|P^{p+1}\right\|=K^{p+1}$ and $\Sigma^{p}$ is a subpolyhedron of $P^{p+1}$. The combination of $f$ in $S^{p}$ and $f_{1}$ at the vertices of $K^{p+1}$ determines a continuous mapping of the subpolyhedron $\Sigma^{p} \cup P^{0}$ of $P^{p}$ into $F_{1}$, of continuous norm less than $\eta_{1}(\epsilon / 2)$. It can therefore be extended to a mapping $g_{1}: P^{p} \rightarrow X$ of continuous norm less than $\epsilon / 2$. Thus $g_{1}$ is a mapping into $U\left(F_{1}, \epsilon / 2\right) \subseteq \mathrm{Cl}\left(U\left(F_{1}, \alpha\right)\right)$. From the definition of $F^{\prime}$ it follows that if $y_{0}=g_{1}\left(c_{0}\right)^{19} g_{1} \mid P^{p-1} \simeq\left[y_{0}\right]$ in $F^{\prime}$. Hence 20 there exists a mapping $g_{2}: P^{p} \rightarrow F^{\prime}$ such that $g_{1} \simeq g_{2}$ in $F^{\prime}$, and $g_{2} \mid P^{p-1}=\left[y_{0}\right]$.

From this point on, the proof that $f \simeq_{\mathrm{e}} 0$ (rel. $\left.c_{0}\right)$ in $F^{\prime}$ proceeds exactly like the remainder of the proof in $\mathrm{H}$ (pp. 484 and 485) that $f \simeq_{\mathbf{c}} 0$ (rel. $\left.x_{0}\right)$ in $U$. The proof of Theorem $4(p)$ is thereby completed.

From the definition of $\zeta$ it is clear that if $\zeta^{\prime}$ and $\eta$ are independent of $x$, so also is $\zeta$. The case $G=X$ is then of most interest, and gives the following theorems.

Theorem 6. If $X$ is $U L C^{1}$ and $u l c^{p}$ it is $U L C^{p}$, if $p \geqq 2$; and if $X$ is $u l c^{0}$ it is $U L C^{0}$.

TheOREM 6.1. ( $p \geqq 0)$. If $X$ is $U L C^{p}$ there exists a positive function $\zeta(\delta)$ such that $U(x, \zeta(\delta))$ is strongly as ${ }^{p}$ rel. $U(x, \delta)$ for all $x$ of $X$.

${ }^{17}$ Lefschetz $[6$, p. 120] $=$ H, Satz (1a). The modifications needed to allow for $X$ being only locally compact are obvious in view of the compactness of $F_{1}$.

18 Continuous norm of $f_{0}=$ least upper bound of $\rho\left(f_{0}(x), f_{0}(y)\right)$ for $x, y$ in the same cell of $\boldsymbol{P}^{p}$.

${ }^{19} c_{0}$ is the point $(1,0, \cdots, 0)$ (cf. $\left.\$ 3\right)$.

${ }^{20} \mathrm{H}$, Satz 2. 


\section{BIBLIOGRAPHY}

1. E. G. Begle, Locally connected spaces and generalised manifolds, Amer. J. Math. vol. 64 (1942) pp. 553-574.

2. - Duality theorems for generalised manifolds, Amer. J. Math. vol. 67 (1945) pp. 59-70.

3. S. Eilenberg and R. L. Wilder, Uniform local connectedness and contractibility, Amer. J. Math. vol. 64 (1942) pp. 613-622.

4. W. Hurewicz, Homotopie, Homologie, und lokaler Zusammenhang, Fund. Math. vol. 25 (1935) pp. 467-485.

5. S. Lefschetz, Topology, Princeton, 1930.

6. - Locally connected and related sets, Ann. of Math. vol. 35 (1934) pp. 118129.

7. - Topics in topology, Annals of Mathematics Studies, 1942, p. 123. 555.

8. R. L. Wilder, Locally connected spaces, Duke Math. J. vol. 1 (1935) pp. 543-

UNIVERSITY OF MANCHESTER 